\title{
Asteroseismology of the $\beta$ Cephei Variable 16 (EN) Lacertae
}

\author{
W.A. Dziembowski \\ Copernicus Astronomical Centre, Polish Academy of Sciences \\ ul. Bartycka 18, 00-716 Warsaw, Poland \\ M. Jerzykiewicz \\ Wroctaw University Observatory \\ ul. Kopernika 11, 51-622 Wroctaw, Poland
}

\begin{abstract}
Various observational data and model calculations are used to identify four pulsation modes detected in this star and to infer its properties. It is concluded that the star's interior must rotate faster than the outer layers.
\end{abstract}

This object is a single-lined spectroscopic binary and an eclipsing variable. It consists of the well-known $\beta$ Cephei star and an invisible secondary. Three circumstances make the $\beta$ Cephei primary exceptionally attractive as a target for asteroseismology: (1) frequencies of as many as four of its pulsation modes are precisely known from time-series observations, (2) multicolour photometry and radial velocity data show that one of the four modes is radial and, of course, which of them it is, and (3) the orbital and eclipse solutions yield a reliable value of the star's mean density.

Frequencies of the four pulsation modes observed in $16 \mathrm{Lac}$ are $f_{1}=5.9112$, $f_{2}=5.8551, f_{3}=5.5033$, and $f_{4}=7.194 \mathrm{c} / \mathrm{d}$ (Jerzykiewicz 1993). The colourto-light and velocity-to-light amplitude ratios show that the $f_{1}$ mode is radial (Jerzykiewicz 1993, Cugier et al. 1994). Mean density of the star amounts to $\langle\rho\rangle=0.0391 \pm 0.0053\langle\rho\rangle_{\odot}$. This value allows us to identity the $f_{1}$ mode as the fundamental, that is, $\mathrm{p}_{1}$.

The measured value of the fundamental mode frequency determines a oneparameter family of stellar models. We assumed standard Population I composition ( $X=0.7, Z=0.02)$ and standard treatment of stellar evolution and pulsations (see Dziembowski \& Pamyatnykh 1993 for details). The range of $\log T_{\text {eff }}$ consistent with various determinations is 4.357 to 4.333 and corresponds to stellar mass range 9 to $10 M_{\odot}$. The range of the star age corresponding to this mass range is 20 to 16 million years and that of the $\mathrm{H}$ abundance in the core, $X_{c}$, is 0.196 to 0.231 .

Considering possible identifications of the $f_{2}, f_{3}$ and $f_{4}$ modes we limited ourselves to modes of spherical harmonic degree $l \leq 2$. We took into account uncertainty of the rotation rate and the azimuthal order of the modes, $m$. Initially we assumed a uniform rotation and we used for model frequencies, $\nu$, the wellknown formula $\nu_{m}=\nu_{0}+m f_{\text {rot }}(1-C)$, where $f_{\text {rot }}=V_{\text {rot }} / 2 \pi R$. The centroid frequencies, $\nu_{0}$, and the Ledoux constants, $C$, were calculated for the models. 
For the surface rotational velocity, $V_{\text {rot }}$, we adopted $40 \mathrm{~km} / \mathrm{s}$. This number is equal to a mean of two published $V_{\text {rot }} \sin i$ values divided by $\langle\sin i\rangle$.

The frequency $f_{2}$ may be identified with the $m=0$ modes whose frequencies are independent of the rotation as long as the effects of centrifugal force are ignored. There are two such possibilities. One is $l=2, p_{0}$. This implies the following parameters: $\log T_{\text {eff }}=4.358, M=9.9 M_{\odot}$. The other is $l=1, g_{1}$, formally implying a mass somewhat above the allowed upper limit of $10 M_{\odot}$. Nevertheless, in view of uncertainties, we regard this identification as tenable. Discrimination between these two $m=0$ modes should be possible with precise measurements of the light and colour phases for the $f_{2}$ term. Closer to the lower mass limit, the $l=1, m=-1, g_{1}$ is also a possibility. More accurate values of $T_{\text {eff }}$ would help discriminate between this and the two previous options.

Neither $f_{3}$ nor $f_{4}$ frequencies may be identified with any of the centroid frequencies in our models. A conservative identification of $f_{4}$ is $l=2, m=-2$, $p_{1}$. This identification requires that the mean value of $f_{\text {rot }}$ is higher than that assumed in the calculated rotational splitting. The required increase ranges from $21 \%$ for the $9 M_{\odot}$ model to $24 \%$ for $10 M_{\odot}$ model. The discrepancy may be due to an underestimate of $V_{\text {rot }}$ or to an inward increase of $f_{\text {rot }}$.

The latter interpretation is supported by two possible identifications of $f_{3}$. The more plausible one seems to be $l=2, m=-2, p_{0}$. It requires a still larger value of the mean $f_{\text {rot }}$. The increase above the adopted value ranges from about $61 \%$ at $10 M_{\odot}$ to $73 \%$ at $9 M_{\odot}$. This mode, being in fact a mixed $\mathrm{p}_{0} / \mathrm{g}_{1}$ mode, samples much more of the star's deep interior than does $p_{1}$. Thus, higher mean $f_{\text {rot }}$ value required implies a significant inward increase of the rotation rate.

The other identification for $f_{3}$ is $l=2, m=2, g_{2}$. This mode samples preferentially the innermost part of the radiative interior where the hydrogen concentration gradient occurs. At $M=9 M_{\odot}$ this identification requires a $63 \%$ increase of $f_{\text {rot }}$ over the adopted value. The required increase growth rapidly with mass and, for instance, at $M=9.5 M_{\odot}$ it is $224 \%$.

Let us point out that we could discriminate between the two identifications of $f_{3}$ if we knew the sign of $m$, but this requires a difficult analysis of the lineprofile observations. However, regardless of which identification is correct, the conclusion that the interior of EN Lac rotates significantly faster than the outer layers is unavoidable within the adopted assumptions.

Acknowledgments. This research was supported by $\mathrm{KBN}$ grants $\mathrm{Nr} 2$ P304 01307 and Nr 2 P304 001 04. MJ gratefully acknowledges a travel grant from the Government of the Republic of South Africa, WAD that from the IAU.

\section{References}

Cugier, H., Dziembowski, W. A., \& Pamyatnykh, A. A. 1994, A\&A, 291, 143 Dziembowski, W. A., \& Pamyatnykh, A. A. 1993, MNRAS, 262, 204 Jerzykiewicz, M. 1993, Acta astr., 43, 13 